\title{
Apparent Diffusion Coefficient Assessment of Brain Development in Normal Fetuses and Ventriculomegaly
}

\author{
Maria Giovanna Di Trani ${ }^{1,2 *}$, Lucia Manganaro ${ }^{3}$, Amanda Antonelli $^{3}$, Michele Guerreri ${ }^{4}$, \\ Riccardo De Feo ${ }^{1,2}$, Carlo Catalano ${ }^{3}$ and Silvia Capuani ${ }^{4}$ \\ ${ }^{1}$ Centro Fermi-Museo Storico della Fisica e Centro Studi e Ricerche Enrico Fermi, Rome, Italy, ${ }^{2}$ SAIMLAL Department, \\ Sapienza University of Rome, Rome, Italy, ${ }^{3}$ Department of Radiological Sciences, Oncology and Anatomo-Pathology, Faculty \\ of Medicine and Dentistry, Sapienza University of Rome, Rome, Italy, ${ }^{4}$ Nuclear Magnetic Resonance and Medical Physics \\ Laboratory, Physics Department, CNR-Institute for Complex Systems, Sapienza University of Rome, Rome, Italy
}

Diffusion neuro-MRI has benefited significantly from sophisticated pre-processing procedures aimed at improving image quality and diagnostic. In this work, diffusion-weighted imaging (DWI) was used with artifact correction and the apparent diffusion coefficient (ADC) was quantified to investigate fetal brain development. The

OPEN ACCESS

Edited by:

Marta Bianciardi,

Massachusetts General Hospital, Harvard Medical School,

United States

Reviewed by:

Robert Mulkern

Boston Children's Hospital, Harvard Medical School, United States Andrea Duggento,

University of Rome Tor Vergata, Italy

*Correspondence:

Maria Giovanna Di Trani mgiovanna.ditrani@gmail.com

Specialty section

This article was submitted to Medical Physics and Imaging,

a section of the journal Frontiers in Physics

Received: 30 April 2019

Accepted: 30 September 2019

Published: 17 October 2019

Citation:

Di Trani MG, Manganaro L, Antonelli A Guerreri M, De Feo R, Catalano $C$ and Capuani S (2019) Apparent Diffusion

Coefficient Assessment of Brain Development in Normal Fetuses and Ventriculomegaly. Front. Phys. 7:160.

doi: 10.3389/fphy.2019.00160
DWI protocol was designed in order to limit the acquisition time and to estimate ADC without perfusion bias. The ADC in normal fetal brains was compared to cases with isolated ventriculomegaly (VM), a common fetal disease whose DWI studies are still scarce. DWI was performed in 58 singleton fetuses (Gestational age (GA) range: 19-38w) at 1.5T. In 31 cases, VM was diagnosed on ultrasound. DW-Spin Echo EPI with $b$-values $=50,200,700 \mathrm{~s} / \mathrm{mm}^{2}$ along three orthogonal axes was used. All images were corrected for noise, Gibbs-ringing, and motion artifacts. The signal-to-noise ratio (SNR) was calculated and the ADC was measured with a linear least-squared algorithm. A multi-way ANOVA was used to evaluate differences in ADC between normal and VM cases and between second and third trimester in different brain regions. Correlation between ADC and GA was assessed with linear and quadratic regression analysis. Noise and artifact correction considerably increased SNR and the goodness-of-fit. ADC measurements were significantly different between second and third trimester in centrum semiovale, frontal white matter, thalamus, cerebellum and pons of both normal and VM brains $(p \leq 0.03)$. ADC values were significantly different between normal and VM in centrum semiovale and frontal white matter ( $p \leq 0.02)$. ADC values in centrum semiovale, thalamus, cerebellum and pons linearly decreased with GA both in normal and VM brains, while a quadratic relation with GA was found in basal ganglia and occipital white matter of normal brains and in frontal white matter of VM $(p \leq 0.02)$. ADC values in all fetal brain regions were lower than those reported in literature where DWI with $b=0$ was performed. Conversely, they were in agreement with the results of other authors who measured perfusion and diffusion contributions separately. By optimizing our DWI protocol we achieved an unbiased quantification of brain ADC in reasonable scan time. Our findings suggested that $A D C$ can be a useful biomarker of brain abnormalities associated with VM.

Keywords: fetal MRI, brain development, DWI, ADC, ventriculomegaly 


\section{INTRODUCTION}

The fascinating process of brain development involves a highly organized mechanism of cell proliferation, histogenesis and myelination leading to the formation of 100 billion neurons in neonatal brains [1].

During gestation, the fetal brain experiences its most vulnerable and important phase of maturation; hence, noninvasive prenatal imaging could be an extremely helpful tool to monitor brain development.

The sensitivity of diffusion-weighted imaging (DWI) techniques in detecting micro-structural changes of cerebral tissues enables the investigation of fetal brain maturation, helping to identify cases of abnormal brain development earlier. Furthermore, the measurement of the apparent diffusion coefficient (ADC) in different fetal brain regions can help to better understand the etiology and the evolution of certain neurological pathologies that develop in fetal age.

One of the most common diseases in fetuses is ventriculomegaly (VM), being diagnosed in approximately 1 out of every 1,000 live births [2], at any gestational age (GA), with a $36.7 \%$ probability of survival without disability [3]. Moreover, VM has been associated with numerous neurological conditions, such as autism [4], epilepsy [5], and schizophrenia [6]; however, DWI studies in fetal brain during gestation are scarce.

An important prognostic factor is whether VM is associated with other abnormalities, since fetuses manifest normal neurological development in the majority of isolated mild VM cases [7]. So far, it is not understood why mild VM can cause motor and cognitive disturbances to the newborn, unlike some cases of severe VMs. For this reason, it is necessary to develop new diagnostic markers to better understand this type of pathology.

The superiority of MRI in identifying fetal brain anomalies, with respect to the sonography [8-10], suggests further investigations on prenatal imaging and DWI is one of the best candidates, given its sensitivity for micro-structural changes.

Prenatal MRI is mainly performed at $1.5 \mathrm{~T}$ to reduce heating effects and potential risks of the radio-frequency exposure [11], which limits image resolution and signal-to-noise ratio (SNR). Another limitation for fetal imaging is artifact due to motion of both the fetus and mother. In small brains, particularly in the second trimester of gestation, low resolution prevents a correct selection of brain regions and therefore the accuracy of measurements, causing ADC values to be biased by partial volume effects.

Studies in fetuses are limited and usually lack an appropriate pre-processing of raw data to improve the quality and reliability of the results [12-15]. In this work, we investigated the potential

\footnotetext{
Abbreviations: DWI, diffusion-weighted imaging; ADC, apparent diffusion coefficient; VM, ventriculomegaly; GA, gestational age; SNR, signal-to-noise ratio; VD, ventricular dilatation; TR, repetition time; TE, echo time; NSA, number of signal averages; IVIM, intra-voxel incoherent motion; WM, white matter; GM, gray matter; ROI, region of interest; CSO, centrum semiovale; FWM, frontal white matter; OWM, occipital white matter; $\mathrm{TH}$, thalamus; $\mathrm{BG}$, basal ganglia; $\mathrm{CH}$, cerebellar hemispheres; SD, standard deviation; RMSE, root mean squared error.
}

ability of DWI denoising methods [16] and artifact corrections $[17,18]$ to assess unbiased ADC values for characterizing normal and VM fetal brain development.

Toward this goal, we used a rapid DWI protocol to measure $\mathrm{ADC}$ in fetal brains at different gestational ages, in the second and third trimester of gestation. With the aim to establish normative ADC measurements, which will be useful to compare and identify cases with anomalies, this study analyzed variations in ADC during gestation of healthy fetuses, compared to cases of fetuses with mild, moderate, and severe VM.

\section{MATERIALS AND METHODS}

DWI was performed in 58 singleton fetuses during the second and third gestational trimester at $1.5 \mathrm{~T}$ (Siemens Avanto, Erlangen, Germany), without mother-fetal sedation.

Thirty-one fetuses presenting ventricular dilatation (VD) on ultrasound investigation were referred to the Radiological Sciences Department of Sapienza University (Rome, Italy) for MRI scans to further assess brain pathologies. Atrial diameter was measured on ultrasound and MRI according to the International Society of Ultrasound in Obstetrics and Gynecology (ISUOG) guidelines [19] and ventriculomegaly (VM) was defined when the atrial diameter of one or both ventricles was $\geq 10 \mathrm{~mm}$. The final cohort consisted of 31 fetuses with isolated VM and 27 normal fetuses, that is, with atrial diameter $<10 \mathrm{~mm}$. In 12 cases, VM was diagnosed as mild ( $\mathrm{VD}<12 \mathrm{~mm}$ ), in 8 as moderate (VD between 12-14 $\mathrm{mm}$ ) and in 10 as severe (VD $\geq 15 \mathrm{~mm}$ ). None of the fetuses showed cerebral anomalies observable with conventional MRI examination, consisting of T2-weighted and FLAIR images.

The study was conducted with the approval of the local Ethics Committee after obtaining a written informed consent from all the mothers. Pregnant women were examined in supine position with a phased-array coil placed over the pelvis. Before performing MRI, a multi-planar scanogram was obtained to localize the fetus.

The Diffusion MRI protocol included a DW-Spin Echo EPI with repetition time $(\mathrm{TR})=4,000 \mathrm{~ms}$, echo time $(\mathrm{TE})=79 \mathrm{~ms}$, bandwidth $=1,628 \mathrm{~Hz} / \mathrm{px}$, matrix dimension $=192 \times 192$, field of view $(\mathrm{FOV})=379 \mathrm{~mm}^{2}$, in-plane resolution $=2 \times 2 \mathrm{~mm}^{2}$, slice-thickness $=4 \mathrm{~mm}$, number of signal averages $(\mathrm{NSA})=2$.

Preliminary tests were performed on three fetuses to carefully choose the diffusion directions and weightings, i.e., $b$-values, of the Diffusion MRI protocol. $B$-values were selected to reduce perfusion effects on ADC estimation and minimize the scanning time to obviate fetal motion. These tests were performed with $b$-values $=0,10,30,50,100,200,400,700 \mathrm{~s} / \mathrm{mm}^{2}$, according to a typical Intra-voxel Incoherent Motion (IVIM) protocol $[20,21]$. We evaluated ADC in a region of white matter (WM) and a region of gray mater (GM) using an IVIM model, monoexponential model with $b$-values $=0,50,200,700 \mathrm{~s} / \mathrm{mm}^{2}$ and mono-exponential model with $b$-values $=50,200,700 \mathrm{~s} / \mathrm{mm}^{2}$. As shown in Figure 1C, the fast decay of the diffusion-weighted signal, mainly due to perfusion, is strongly reduced at $b$-value $=$ $50 \mathrm{~s} / \mathrm{mm}^{2}$. Therefore, from the comparison between diffusion parameters estimated using IVIM and mono-exponential 
models, we selected $b$-values $=50,200,700 \mathrm{~s} / \mathrm{mm}^{2}$ for the diffusion protocol used to acquire data from all subjects of the healthy and VM cohorts. The total scan time was approximately $2 \mathrm{~min}$.

\section{PRE-PROCESSING AND DATA ANALYSIS}

After a first inspection, 2 normal and 11 VM cases (2 mild, 2 moderate, 7 severe) showed motion artifacts impossible to correct or severe ventricle dilatation that prevented correct ROI selection; thus 13 subjects were discarded from the following analysis.

The pipeline for image correction (Figure 1) started with noise removal by using dwidenoise tool [16]; then, unring [17] was used to correct denoised DWI for Gibbs-ringing artifacts. Eventually, images were corrected for fetal/mother motion artifacts by using FLIRT [18]: each volume was registered to the image at $b$-value $=50 \mathrm{~s} / \mathrm{mm}^{2}$ through a rigid-body transformation, using a tri-linear interpolation method.

Seven regions of interest (ROI) were manually placed in the centrum semiovale (CSO), frontal white matter (FWM), occipital white matter (OWM), thalamus $(\mathrm{TH})$, basal ganglia (BG), cerebellar hemispheres $(\mathrm{CH})$ and pons, as reported in Figure 1B. In some VM cases, ROIs could not be placed in CSO (3 cases), FWM (3), BG (3), TH (3), OWM (3), CH (1) because $\mathrm{VM}$ was severe or the brain was too small.

SNR of raw and corrected DWI at each $b$-value was calculated to evaluate the reliability of estimated ADC values. SNR was calculated in each volume as the ratio between DWI signal averaged over all brain ROIs and the standard deviation (SD) of noise, as follows:

$$
\text { SNR }=0.655 \frac{\text { mean }(\text { signal })}{S D(\text { noise })}
$$

where noise was estimated in the background and the factor 0.655 was used to account for Rayleigh distribution of background noise [22].

$\mathrm{ADC}$ values were measured through a fitting procedure developed in MATLAB, with a linear least-squares algorithm, and the goodness-of-fit was evaluated with the root mean squared error (RMSE). The fitting procedure was carried out voxel-byvoxel, making inter-volume motion correction a mandatory step for voxel alignment.

A multi-way ANOVA with Bonferroni correction was used to evaluate differences in ADC between normal and VM cases and between the second and third TR.

The relationship between ADC measurements and GA was assessed with linear and quadratic regression analysis by calculating the adjusted coefficient of determination $R^{2}$ and the $b$-value for the Fisher-test to evaluate the statistical significance of the model. Initially, linear regression was performed in each ROI. However, we observed that some brain regions (FWM, OWM and BG) showed an evident non-linear dependence on GA, thus we performed a quadratic regression in those regions. Outliers, that is values bigger than three median absolute deviations away from median, were not considered in the regression analysis.

\section{RESULTS}

The preliminary tests, performed to choose the optimal $b$-values of the Diffusion MRI protocol for reducing perfusion effects on ADC measurements, showed a fast decay of the DWI signal due to perfusion at low $b$-values $\left(b \leq 50 \mathrm{~s} / \mathrm{mm}^{2}\right)$. However, the fast signal decay was strongly reduced at $b$-values $=50 \mathrm{~s} / \mathrm{mm}^{2}$ (Figure 1C). Therefore we performed the study using $b$-values equal to $50,200,700 \mathrm{~s} / \mathrm{mm}^{2}$. Moreover, the ADC estimated by the mono-exponential function with three $b$-values did not differ more than the SD the from the diffusion coefficient derived by the only diffusion component of the IVIM model, estimated with eight $b$-values.

SNR and the quality of DW-images considerably increased after noise and artifact correction $\left(\mathrm{SNR}_{\text {corr }}=19.5 \pm 7.8\right.$, $\mathrm{SNR}_{\text {raw }}=7.8 \pm 3.1$; both calculated at $b$-value $=700 \mathrm{~s} / \mathrm{mm}^{2}$ ), as shown in Figure 2A. Also the goodness-of-fit significantly increased after correction RMSE $_{\text {raw }}=0.07, \mathrm{RMSE}_{\mathrm{corr}}=0.06$, $p=0.0001$, Figure 2B).

Mean ADC values in normal and VM fetuses, obtained in the second and third trimester of gestation and in different cerebral regions, are shown in Figure 3. Significant differences were found between second and third trimester in $\mathrm{CSO}, \mathrm{FWM}, \mathrm{TH}, \mathrm{CH}$ and pons of both normal and VM brains $(p \leq 0.03$ ). ADC values in normal and VM ROIs were not significantly different, except for $\mathrm{CSO}$ and $\mathrm{FWM}(p \leq 0.02)$.

Linear regression analysis showed ADC values in CSO, TH, $\mathrm{CH}$ and pons to significantly decrease with GA both in normal $(p \leq 0.001$; Figures 4A,B,D,E and Table 2) and VM brains ( $p$ $\leq 0.001$; Figures 4G,H,J,K and Table 2 ). A significant quadratic relationship with GA was found in BG and OWM of normal brains ( $p \leq 0.02$; Figures 4C,F and Table 2) and FWM of VM cases ( $p \leq 0.02$; Figure 4 I and Table 2).

In the entire data set, 6 outliers were found in TH $(1.68 \times$ $\left.10^{-3} \mathrm{~mm}^{2} / \mathrm{s}\right)$, BG $\left(1.77,1.69 \times 10^{-3} \mathrm{~mm}^{2} / \mathrm{s}\right)$, OWM $\left(2.68 \times 10^{-3}\right.$ $\left.\mathrm{mm}^{2} / \mathrm{s}\right)$ and $\mathrm{CH}\left(1.74 \times 10^{-3} \mathrm{~mm}^{2} / \mathrm{s}\right)$ for normal brains and in pons $\left(1.49 \times 10^{-3} \mathrm{~mm}^{2} / \mathrm{s}\right)$ for VM brains; these values were discarded from both linear and quadratic regression analysis.

\section{DISCUSSIONS AND CONCLUSIONS}

In this work, prenatal DWI was performed at $1.5 \mathrm{~T}$ and the $\mathrm{ADC}$ was measured in fetal brains during the second and third trimester of gestation, both in normal and VM cases.

Prenatal imaging could be strongly affected by uncontrollable fetal/mother movement, especially when performed without sedation. In order to limit motion-derived artifacts, we designed our DWI protocol with limited number of signal averages (NSA $=2)$ to be as fast as possible, causing poor $\operatorname{SNR}\left(\operatorname{SNR}_{\text {raw }}=\right.$ $7.8 \pm 3.1$ at $\mathrm{b}=700 \mathrm{~s} / \mathrm{mm}^{2}$ ) in our data. To overcome image quality limitations, raw data were denoised and corrected for motion and Gibbs' ringing artifacts. Results, reported in Figure 2A, showed that image pre-processing significantly improved SNR and goodness-of-fit, guaranteeing a better ROI selection of different fetal brain regions and a reliable estimation of the ADC. Moreover, volume registration was fundamental to 


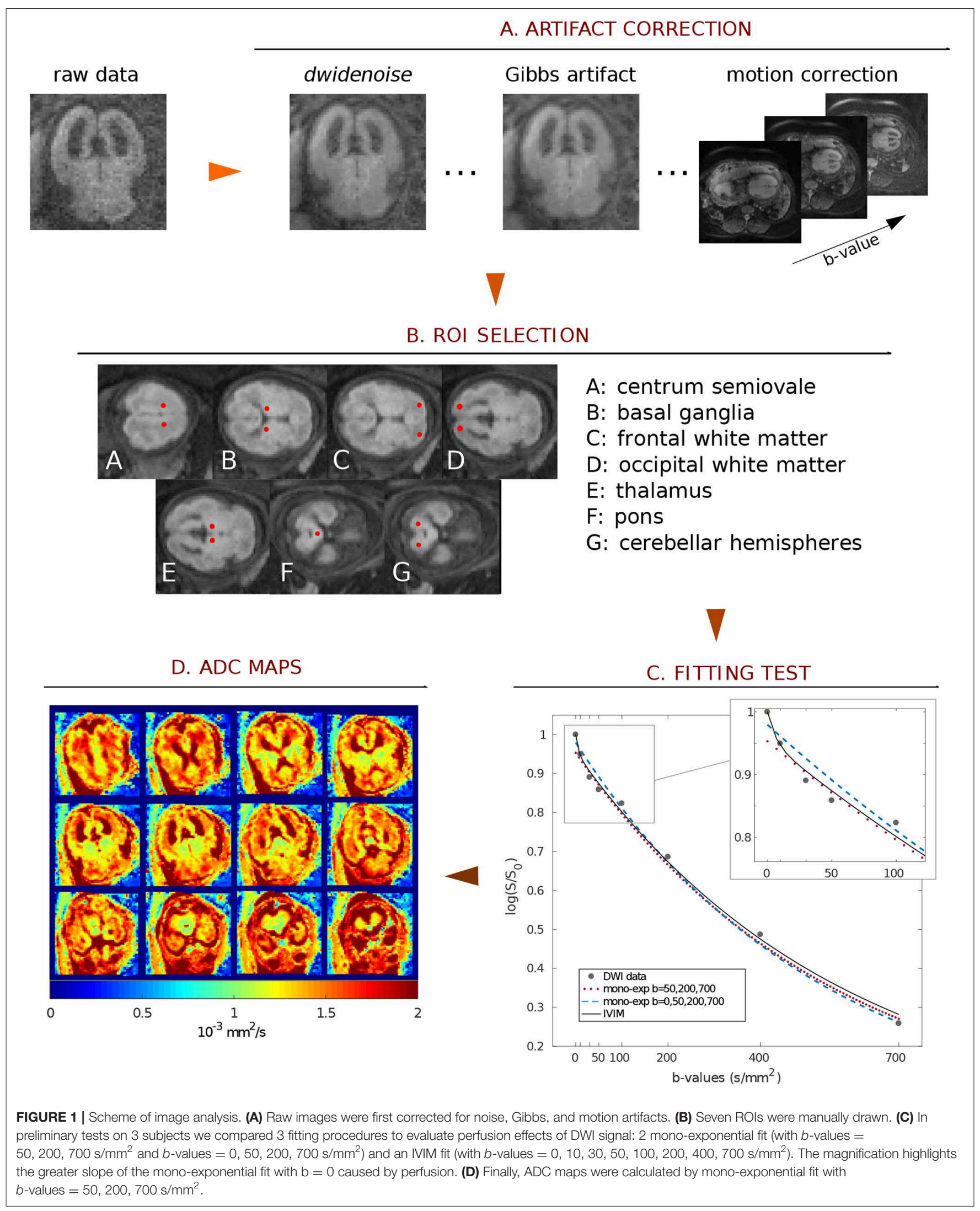



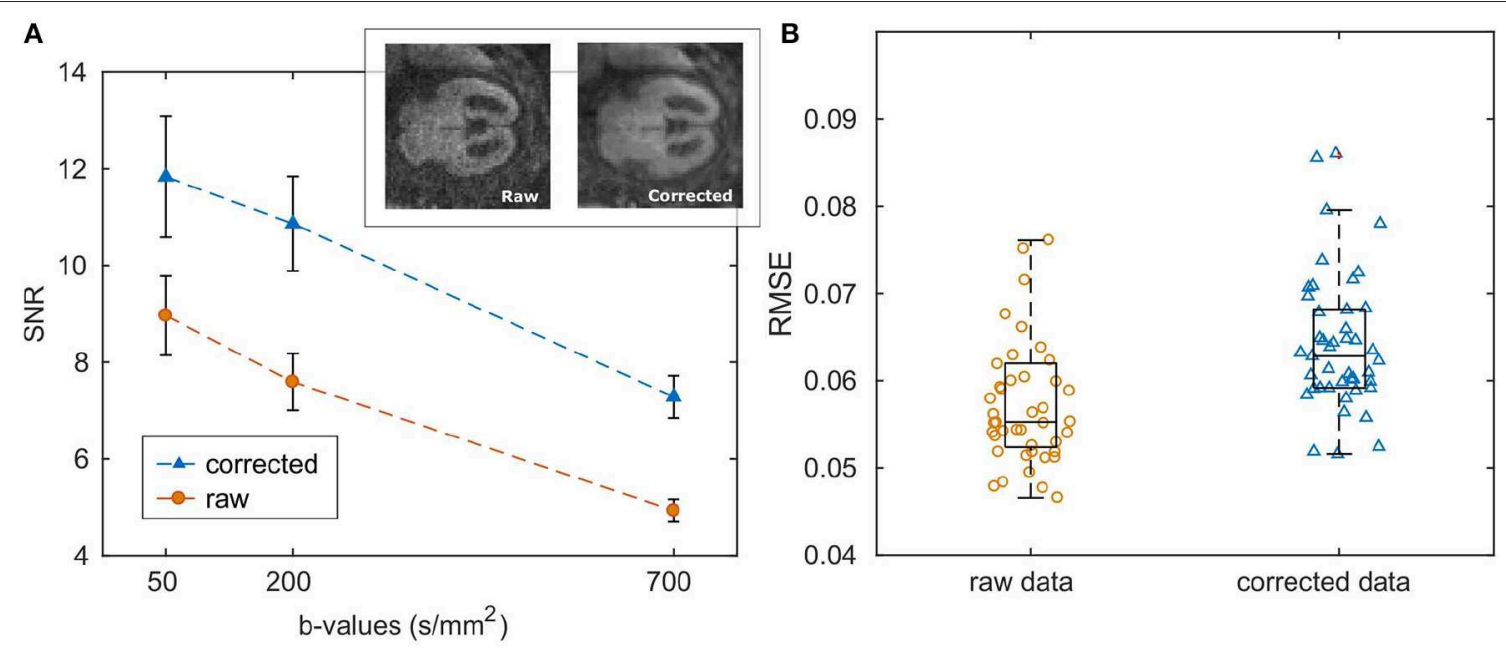

FIGURE 2 | (A) Mean and standard error of SNR calculated over the entire patient cohort. An example of raw and corrected DWI at $b$-value $=700 \mathrm{~s} / \mathrm{mm}^{2}$ is reported in the top right corner of the plot. (B) Boxplots of the root mean squared error of ADC fit; dots represent RMSE for each subject averaged over the ROI.

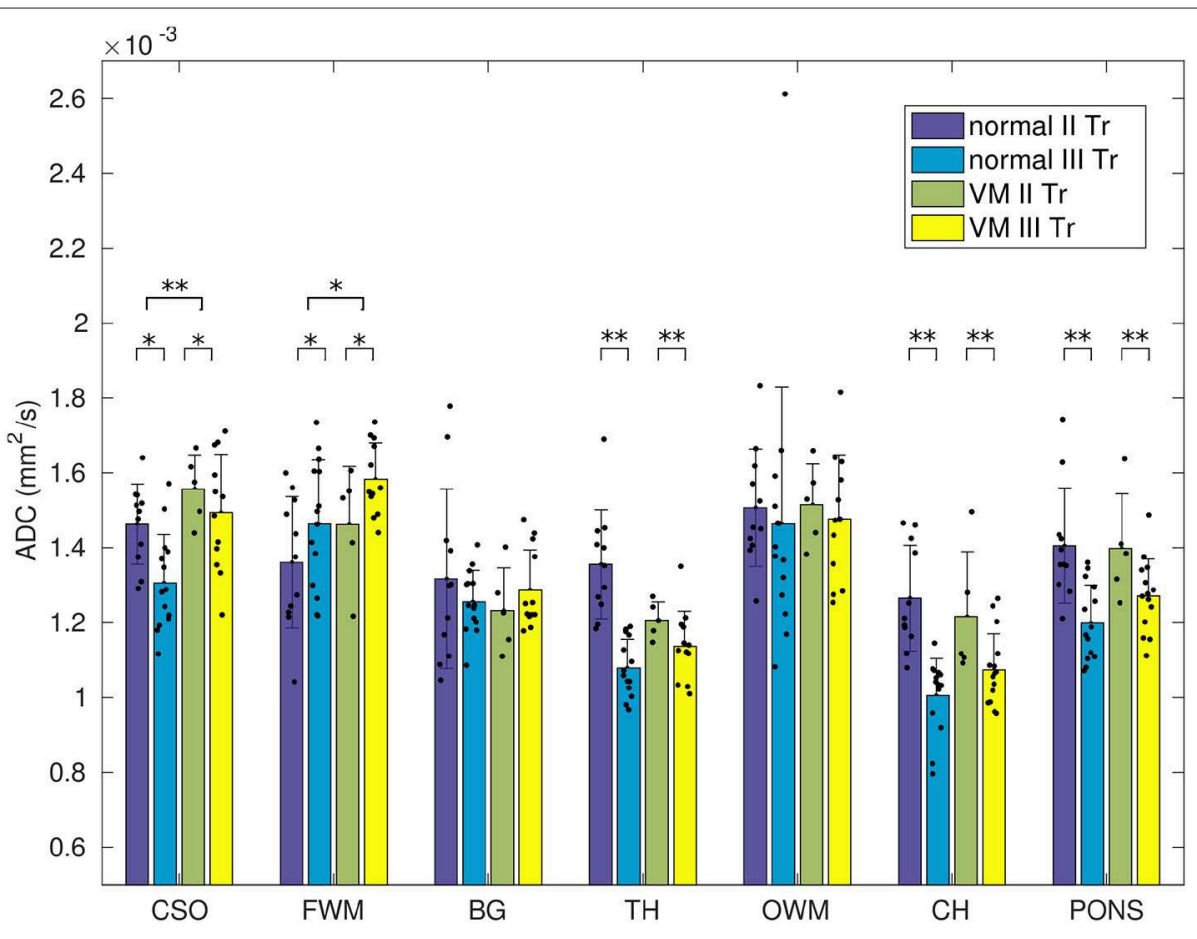

FIGURE 3 | ADC measurements in normal and VM brains of fetuses in the second and third trimester of gestation. Dots represent the ADC calculated for each subject and asterisks represent the $p$-value of multi-way ANOVA $\left({ }^{*} p<0.02 ;{ }^{* *} p<10^{-3}\right.$ ).

minimize voxel misalignment, since the fitting procedure was carried-out voxel-by-voxel.

Since ADC estimation is strongly influenced by the diffusion weighting, in this study $b$-values were carefully chosen in order to minimize perfusion contamination of ADC measurements; therefore, DWI with $b$-value $=50 \mathrm{~s} / \mathrm{mm}^{2}$ was acquired as first volume and used to normalize data prior to fit. It is well known that DWI with low diffusion weighting is sensitive to perfusion motion, resulting from blood micro-circulation in capillaries [20], which contributes to increased ADC values. By normalizing diffusion data with the volume at $b$-value $=50 \mathrm{~s} / \mathrm{mm}^{2}$, we reduced the contribution of perfusion in our measurements, finding, in normal fetuses, lower ADC values compared to other studies that used $\mathrm{b}=0[12-15]$.

In a recent study [21], an IVIM technique was carried out to separately measure the contribution of perfusion and diffusion, 


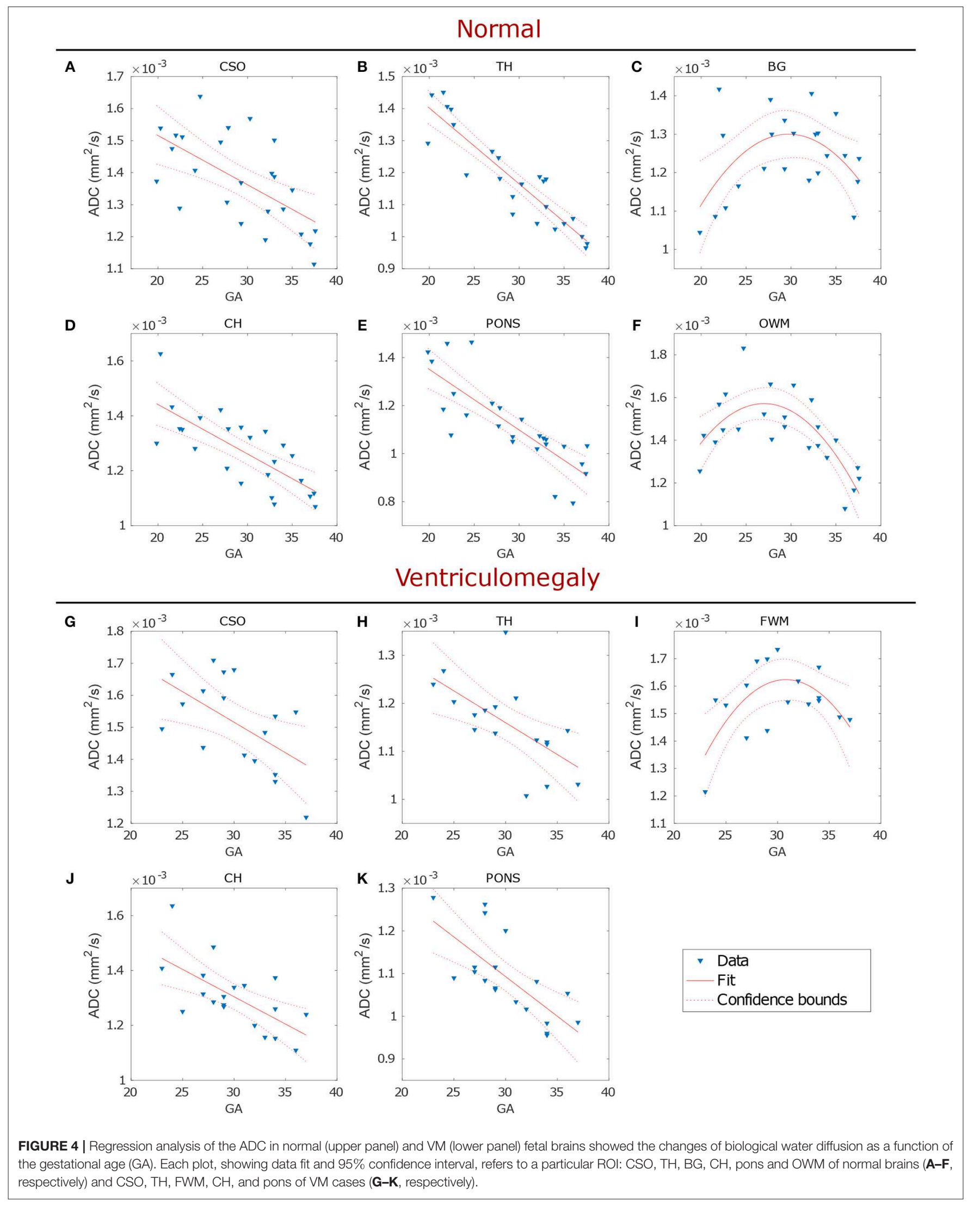


in developing fetal brain. The authors [21] measured lower ADC values in $\mathrm{CSO}, \mathrm{FWM}, \mathrm{OWM}, \mathrm{CH}$ and pons compared to those reported in literature [12-15], demonstrating that perfusion motion can affect ADC measurements in fetal brains. Our ADC values were in good accordance with the measurements derived from the contribution of solely diffusion in the IVIM study [21], suggesting that our acquisition strategy effectively minimized perfusion effects.

However, authors [21] acquired DWI with nine $b$-values (from 0 to $800 \mathrm{~s} / \mathrm{mm}^{2}$ ) in order to fit the IVIM model, using a total acquisition time of about $5 \mathrm{~min}$. As a result of a longer acquisition time, compared to that used in this work ( $2 \mathrm{~min}$ ), the IVIM study [21] discarded $56 \%$ of subjects due to fetal movements, while in this study only $22 \%$ of subjects was rejected for motion artifacts.

Analogously to other works in normal fetuses [13-15, 21, 23], we found that during brain development, ADC linearly decreased in $\mathrm{CSO}, \mathrm{TH}, \mathrm{CH}$ and pons, as well as in VM cases, reflecting the morphological changes of brain maturation that involves increasing cellularity, histogenesis and myelination [24]. The latter starts in the spinal cord (12 GA), followed by pons, cerebellar peduncles, and thalamic axons (14-20 GA), in the frontal, parietal and occipital lobes (30 GA) and finally in the cortex (35 GA), lasting for decades in the human brain [2528]. The process of myelination begins with the accumulation of lipids and proteins [29] and the proliferation of oligodendrocyte precursors [30], causing a reduction of extracellular space, with a consequent decrease in $\mathrm{ADC}$ values. According to previous results $[13-15,21,23]$, the $\mathrm{ADC}$ was found to be significantly higher in the fetal white matter than in the deep brain nuclei (BG, TH, and pons), suggesting an immature status of fiber myelination during fetal development.

Consistently with the timeline of myelination, we found the decrease of diffusivity in OWM (normal cases) and FWM (VM cases) to start from 28-30 GA, later than $\mathrm{CSO}, \mathrm{TH}, \mathrm{CH}$ and pons. An initial increase in ADC values was measured in cortical WM, from 23 to $30 \mathrm{GA}$, followed by a decline after $30 \mathrm{GA}$, as reported in other studies [31-33]. These results are in agreement with the normal progression of myelination that occurs from central to peripheral brain.

Although in VM fetuses no other alteration was reported by ultrasound exam, we found significantly higher diffusivity (Figure 3 and Table 1) in CSO and FMW compared to normal fetal brain. This result may reveal delayed or abnormal maturation of white matter, as reported in other studies in neonates with isolated mild VM [34-36], suggesting that the ADC in CSO and FWM could be a marker of altered development and disorders associated with VM.

Furthermore, regression analysis in $\mathrm{TH}$ showed that ADC values were more strongly correlated with GA in normal than in VM cases (Table 2 and Figure 4B), while no significant correlation was found in OWM of VM cases, suggesting the possibility of a delayed white matter maturation in VM brains, especially during the third trimester of gestation.

In contrast to a recent work [12], we did not find significantly higher ADC values in BG of VM compared to normal fetuses. Anatomically, BG is the nearest structure to the lateral ventricles, hence it is more prone to be affected by signal contamination
TABLE 1 | Multi-way ANOVA to investigate the effects of brain maturation and VM disease on ADC measurements.

\begin{tabular}{lcc}
\hline & II vs. III TR & Normal vs. VM \\
\hline CSO & 0.005 & $<10^{-3}$ \\
FWM & 0.03 & 0.02 \\
BG & $\mathrm{ns}$ & $\mathrm{ns}$ \\
TH & $<10^{-4}$ & $\mathrm{~ns}$ \\
OWM & $\mathrm{ns}$ & $\mathrm{ns}$ \\
PONS & $<10^{-4}$ & $\mathrm{~ns}$ \\
$\mathrm{CH}$ & $<10^{-4}$ & $\mathrm{~ns}$ \\
\hline
\end{tabular}

TABLE 2 | Regression analysis between ADC and gestational age.

\begin{tabular}{|c|c|c|c|c|c|c|c|c|}
\hline & \multicolumn{4}{|c|}{ Normal } & \multicolumn{4}{|c|}{ VM } \\
\hline & \multicolumn{2}{|c|}{ Linear } & \multicolumn{2}{|c|}{ Quadratic } & \multicolumn{2}{|c|}{ Linear } & \multicolumn{2}{|c|}{ Quadratic } \\
\hline & $R^{2}$ & $\mathbf{p}$ & $R^{2}$ & p & $R^{2}$ & $\mathbf{p}$ & $R^{2}$ & p \\
\hline CSO & 0.36 & 0.001 & - & - & 0.28 & 0.02 & - & - \\
\hline FWM & - & ns & - & ns & - & ns & 0.34 & 0.02 \\
\hline$B G$ & - & ns & 0.19 & 0.04 & - & ns & - & - \\
\hline $\mathrm{TH}$ & 0.82 & $<10^{-5}$ & - & - & 0.36 & 0.006 & - & - \\
\hline OWM & 0.17 & 0.02 & 0.54 & 0.0001 & - & ns & - & ns \\
\hline PONS & 0.54 & $<10^{-3}$ & - & - & 0.48 & $<10^{-2}$ & - & - \\
\hline $\mathrm{CH}$ & 0.65 & $<10^{-4}$ & - & - & 0.38 & 0.003 & - & - \\
\hline
\end{tabular}

from ventricles due to volume misalignment. This could have caused the disagreement with the work of Yaniv et al. [12], since they did not use any method to realign DWI.

Moreover, the same authors [12] found lower ADC in FWM of VM fetuses compared to normal fetal brain, whereas we obtained the opposite result (Figure 3). FWM and OWM regions are supplied by the carotid system with large vessels, so perfusion is relatively high, as shown in reference [21]. We suspect that ADC measured by Yaniv et al. [12] was partially affected by perfusion, since they acquired data using $b$-values $=0$ and $700 \mathrm{~s} / \mathrm{mm}^{2}$, leading to the discrepancies with our results.

To the best of our knowledge, no other works have investigated the ADC in brain development of VM fetuses (Figures 4G-K). Although our study was limited by a small statistical sample, it could help to establish criteria for recognizing abnormal brain development that is important for early diagnosis of diseases.

In addition to the small number of subjects, our study was also limited by the slice thickness, which should be reduced to minimize partial volume effects and to enable more accurate identification of brain tissues.

Our study on VM brains revealed the sensitivity of DWI in brain diseases, making this technique suitable also to patient follow-up. Moreover, our routine image correction had proven to be a reasonable and fast method for improving the quality of diffusion-weighted images and for providing a reliable identification of ROIs.

Perfusion motion might affect DWI signal, causing ADC to be artificially higher; in this work we showed that, using $b$-value $=$ 
$50 \mathrm{~s} / \mathrm{mm}^{2}$ as first acquired diffusion-weighted volume, the ADC was quantified without significant perfusion contamination, since our measurements were comparable to those obtained by the IVIM model [21].

Although our work is limited by the use of 1.5T magnetic field, our rapid acquisition protocol combined with artifact correction allowed a reliable estimation of the ADC in a very short time and the identification of anomalies in fetal brain development.

This work suggested that regional differences in fetal ADC values and their variations with GA reflect micro-structural and physiological changes due to brain maturation. These preliminary data can contribute to delineate a reference standard to assess the normal progress of sulcation and myelination as well as the normative biometry of the fetal brain, contributing to improve knowledge of brain maturation.

\section{DATA AVAILABILITY STATEMENT}

The datasets generated for this study are available on request to the corresponding author.

\section{REFERENCES}

1. Ackerman S. (1992). Discovering the Brain. Washington, DC: National Academies Press.

2. Pilu G, Perolo A, Falco P, Visentin A, Gabrielli S, Bovicelli L. Ultrasound of the fetal central nervous system. Curr Opin Obstet Gynecol. (2000) 12:93-103. doi: 10.1097/00001703-200004000-00007

3. Carta S, Kaelin Agten A, Belcaro C, Bhide A. Outcome of fetuses with prenatal diagnosis of isolated severe bilateral ventriculomegaly: systematic review and meta-analysis. Ultrasound Obstet Gynecol. (2018) 52:165-73. doi: 10.1002/uog.19038

4. Palmen SJ, Hulshoff Pol HE, Kemner C, Schnack HG, Durston S, Lahuis BE, et al. Increased gray-matter volume in medication-naive high-functioning children with autism spectrum disorder. Psychol Med. (2005) 35:561-70. doi: 10.1017/S0033291704003496

5. Jackson DC, Irwin W, Dabbs K, Lin JJ, Jones JE, Hsu DA, et al. Ventricular enlargement in new-onset pediatric epilepsies. Epilepsia. (2011) 52:2225-32. doi: 10.1111/j.1528-1167.2011.03323.x

6. Wright IC, Sham P, Murray RM, Weinberger DR, Bullmore ET. Genetic contributions to regional variability in human brain structure: methods and preliminary results. Neuroimage. (2002) 17:256-71. doi: 10.1006/nimg.2002.1163

7. Huisman TA. Fetal magnetic resonance imaging of the brain: is ventriculomegaly the tip of the syndromal iceberg? Semin Ultrasound CT MR. (2011) 32:491-509. doi: 10.1053/j.sult.2011. 08.002

8. Girard NJ. Magnetic resonance imaging of fetal developmental anomalies. Top Magn Reson Imaging. (2011) 22:11-23. doi: 10.1097/RMR.0b013e3182425bb2

9. Manganaro L, Bernardo S, La Barbera L, Noia G, Masini L, Tomei A, et al. Role of foetal MRI in the evaluation of ischaemic-haemorrhagic lesions of the foetal brain. J Perinat Med. (2012) 40:419-26. doi: 10.1515/jpm-2011-0182

10. Yin S, Na Q, Chen J, Li-Ling J, Liu C. Contribution of MRI to detect further anomalies in fetal ventriculomegaly. Fetal Diagn Ther. (2010). 27:20-4. doi: $10.1159 / 000260389$

11. Weisstanner C, Gruber GM, Brugger PC, Mitter C, Diogo MC, Kasprian G, et al. Fetal MRI at 3T-ready for routine use? Br J Radiol. (2016) 90:20160362. doi: 10.1259/bjr.20160362

12. Yaniv G, Katorza E, Bercovitz R, Bergman D, Greenberg G, Biegon A, et al. Region-specific changes in brain diffusivity in fetal isolated mild ventriculomegaly. Eur Radiol. (2016) 26:840-8. doi: 10.1007/s00330-015-3893-y

\section{ETHICS STATEMENT}

This study was carried out in accordance with the recommendations of the International Society of Ultrasound in Obstetrics and Gynecology (ISUOG) guidelines with written informed consent from all subjects. All subjects gave written informed consent in accordance with the Declaration of Helsinki. The protocol was approved by the Policlinico Umberto I of Sapienza University Ethics Committee.

\section{AUTHOR CONTRIBUTIONS}

$\mathrm{MD}, \mathrm{SC}$, and LM conceived the original idea and planned the experiments. AA, LM, and SC carried out the experiment. MD elaborated all data and performed statistical analysis with contribution of $\mathrm{MG}$ and $\mathrm{RD}$. $\mathrm{MD}$ and $\mathrm{SC}$ wrote the manuscript with contribution of LM. AA, CC, LM, and SC supervise the project. All authors contributed to the interpretation of the results, read, and approved the final manuscript.

13. Hoffmann C, Weisz B, Lipitz S, Yaniv G, Katorza E, Bergman D, et al. Regional apparent diffusion coefficient values in 3rd trimester fetal brain. Neuroradiology. (2014) 56:561-7. doi: 10.1007/s00234-014-1359-6

14. Schneider MM, Berman JI, Baumer FM, Glass HC, Jeng S, Jeremy RJ, et al. Normative apparent diffusion coefficient values in the developing fetal brain. AJNR Am J Neuroradiol. (2009) 30:1799-803. doi: 10.3174/ajnr.A1661

15. Han R, Huang L, Sun Z, Zhang D, Chen X, Yang X, et al. Assessment of apparent diffusion coefficient of normal foetal brain development from gestational age week up to term age: a preliminary study. Foetal Diagn Ther. (2014) 37:102-7. doi: 10.1159/000363650

16. Veraart J, Novikov DS, Christiaens D, Ades-aron B, Sijbers J, Fieremans E. Denoising of diffusion MRI using random matrix theory. NeuroImage. (2016) 142:394-406. doi: 10.1016/j.neuroimage.2016.08.016

17. Kellner E, Dhital B, Kiselev VG, Reisert M. Gibbs-ringing artifact removal based on local subvoxel-shifts. Magnet Res Med. (2016) 76:1574-81. doi: 10.1002/mrm.26054

18. Jenkinson M, Beckmann CF, Behrens TE, Woolrich MW, Smith SM. FSL. NeuroImage. (2012) 62:782-90. doi: 10.1016/j.neuroimage.2011.09.015

19. International Society of Ultrasound in Obstetrics and Gynecology (ISUOG). Sonographic examination of the fetal central nervous system for performing the 'basic examination' and the 'fetal neurosonogram'. Ultrasound Obstet Gynecol. (2007) 29:109-16. doi: 10.1002/uog.3909

20. Le Bihan D, Breton E, Lallemand D, Aubin ML, Vignaud J, Laval-Jeantet M. Separation of diffusion and perfusion in intravoxel incoherent motion MR imaging. Radiology. (1988) 168:497-505.

21. Yuan X, Yue C, Yu M, Chen $\mathrm{P}, \mathrm{Du} \mathrm{P}$, Shao $\mathrm{CH}$, et al. Fetal brain development at 25-39 weeks gestational age: a preliminary study using intravoxel incoherent motion diffusion-weighted imaging. J Magn Reson Imaging. (2019) 50:899-909. doi: 10.1002/jmri.26667

22. Dietrich O, Raya JG, Reeder SB, Reiser MF, Schoenberg SO. Measurement of signal-to-noise ratios in MR images: influence of multichannel coils, parallel imaging, and reconstruction filters. J Magn Reson Imaging. (2007) 26:375-85. doi: 10.1002/jmri.20969

23. Brouwer MJ, van Kooij BJ, van Haastert IC, Koopman-Esseboom C, Groenendaal F, de Vries LS, et al. Sequential cranial ultrasound and cerebellar diffusion weighted imaging contribute to the early prognosis of neurodevelopmental outcome in preterm infants. PLoS ONE. (2014) 9:e109556. doi: 10.1371/journal.pone.0109556

24. Langworthy OR. Development of behavior patterns and myelinization of the nervous system in the human fetus and infant. Contrib Embryol. (1993) 139:1-57. 
25. Miller JH, McKinstry RC, Philip JV, Mukherjee P, Neil JJ. Diffusiontensor MR imaging of normal brain maturation: a guide to structural development and myelination. Am J Roentgenol. (2003) 180:851-9. doi: 10.2214/ajr.180.3.1800851

26. Bird CR, Hedberg M, Drayer BP, Keller PJ, Flom RA, Hodak JA. MR assessment of myelination in infants and children: Usefulness of marker sites. AJNR Am J Neuroradiol. (1989) 10:731-40.

27. Barkovich AJ, Kjos BO, Jackson DE Jr, Norman D. Normal maturation of the neonatal and infant brain: MR imaging at 1.5T. Radiology. (1988) 166:173-80.

28. Hayakawa K., Konishi Y, Kuriyama M, Konishi K, Matsuda T. Normal brain maturation in MRI. Eur J Radiol. (1990) 12:208-15.

29. Gilles FH, Shankle W, Dooling EC. Myelinated tracts: growth patterns. In: Gilles FH, Leviton A, Dooling EC, eds. The Developing Human Brain-Growth and Epidemiologic Neuropathology. Boston: John Wright PSG Inc. (1983). p. 117-83.

30. Back SA, Luo NL, Borenstein NS, Volpe JJ, Kinney HC. Arrested oligodendrocyte lineage progression during human cerebral white matter development: dissociation between the timing of progenitor differentiation and myelinogenesis. J Neuropathol Exp Neurol. (2002) 61:197-211. doi: 10.1093/jnen/61.2.197

31. Schneider JF, Confort-Gouny S, Le Fur Y, Viout P, Bennathan M, Chapon F, et al. Diffusion-weighted imaging in normal fetal brain maturation. Eur Radiol. (2007) 17:2422-9. doi: 10.1007/s00330-007-0 634-x

32. Chau V, Synnes A, Grunau RE, Poskitt KJ, Brant R, Miller SP. Abnormal brain maturation in preterm neonates associated with adverse developmental outcomes. Neurology. (2013) 81:2082-9. doi: 10.1212/01.wnl.0000437298.43688.b9
33. Hagmann $\mathrm{P}$, Sporns $\mathrm{O}$, Madan $\mathrm{N}$, Cammoun $\mathrm{L}$, Pienaar R, Wedeen VJ, et al. White matter maturation reshapes structural connectivity in the late developing human brain. Proc Natl Acad Sci USA. (2010) 107:19067-72. doi: 10.1073/pnas.10090 73107

34. Gilmore JH, Smith LC, Wolfe HM, Hertzberg BS, Smith JK, Chescheir NC, et al. Prenatal mild ventriculomegaly predicts abnormal development of the neonatal brain. Biol Psychiatry. (2008) 64:1069-76. doi: 10.1016/j.biopsych.2008.07.031

35. Goodlett CB, Fletcher PT, Gilmore JH, Gerig G. Group analysis of DTI fiber tract statistics with application to neurodevelopment. Neuroimage. (2009) 45:133-42. doi: 10.1016/j.neuroimage.2008.10.06

36. Lockwood Estrin G, Kyriakopoulou V, Makropoulos A, Ball G, Kuhendran L, Chew A, et al. Altered white matter and cortical structure in neonates with antenatally diagnosed isolated ventriculomegaly. Neuroimage Clin. (2016) 11:139-48. doi: 10.1016/j.nicl.2016.01.012

Conflict of Interest: The authors declare that the research was conducted in the absence of any commercial or financial relationships that could be construed as a potential conflict of interest.

Copyright (C) 2019 Di Trani, Manganaro, Antonelli, Guerreri, De Feo, Catalano and Capuani. This is an open-access article distributed under the terms of the Creative Commons Attribution License (CC BY). The use, distribution or reproduction in other forums is permitted, provided the original author(s) and the copyright owner(s) are credited and that the original publication in this journal is cited, in accordance with accepted academic practice. No use, distribution or reproduction is permitted which does not comply with these terms. 